\title{
CHEMICAL ENGINEERING : A THRUST ON RESEARCH \& TECHNOLOGY
}

By

Jaydip Datta

\section{ABSTRACT :}

In this Review we will point out some Research \& Technological view highlighting the following areas process modelling, simulation \& optimisation, environmental pollution, and computational fluid dynamics.

[1 ] CHEMICAL PROCESS TECHNOLOGY - Synthesis involving technological aspects on the chemical process as well as feasibility study. It is also known as process modelling / simulation \& optimization (https://www.researchgate.net/post/ls there any basic difference between Chemistry an d Chemical Engineering ).

(https://www.researchgate.net/post/What is the basic difference between Engineering a nd Technology ).

[2] ENVIRONMENTAL ENGINEERING - Environmental Science including Lead \& Arsenic pollution as well as Toxicological effect including biotechnology .( https://www.researchgate.net/post/Which Car having more Lead automobile emission either Petrol Diesel )

(https://www.researchgate.net/post/What is the application of NON-

DISPERSIVE IR spectroscopy )

(https://www.researchgate.net/post/What is the utility of DRAEGERS Tube in Enviro nmental Pollution)

[ 3 ] COMPUTATIONAL FLUID DYNAMICS : It is the most popular research area w.r.t pure Chemical Engineering research including Hydrodynamics.( https://www.researchgate.net/post/FORTRAN vs C a comperative approach ) (https://www.researchgate.net/post/ls Best Fit Hydrodynamic Study is also an Interdisci plinary part of CFD ). 


\section{REFERENCES :}

1.JAYDIP DATTA, MOLECULAR CFD : AN ALTERNATIVE APPROACH TO HYDRODYNAMIC MODELLING ,July 2019 ,DOI: 10.13140/RG.2.2.29747.78886/7

(https://www.researchgate.net/publication/334771118 MOLECULAR CFD AN ALTERNAT IVE APPR OACH TO HYDRODYNAMIC MODELLING? sg=Ujm0mIHt3X0EQ-3n6J9scVLQ2rnVgbccvTPsLxCYi7nQDIkY FtoT9y4W8Uyw5AXOcNhFL4HECFjp1hr1RYoc3y0 o8YovKieK8iY iF.u3cRnD-

vUZ7cbSj5YNeR5sQOFNackl 18UPF7SfbV5KcH g9chdlnglEluj7wKS LZmA8ZztxP0hY2Q $\underline{3 \mathrm{YHkG6Q})}$

2.JAYDIP DATTA at al, BIO-TECHNOLOGY: Newer Biology -An Impression ,March 2020 ,DOI: $10.13140 /$ RG.2.2.22829.61925 (https://www.researchgate.net/publication/339935767 BIOTECHNOLOGY Newer Biology An Impression? sg=rw60gkx5Y0C8jshVDQbv9nTp8QkIDHn2ZR FnA5WYYOYUd2 kWoEs9bbShoY7o9WCMULUsyAbSZyZ9mIL5UEHRaqX4cWp2lpZ9eq0CgoC.kXP KQbeltTD1aq90MG8StEDaBpF3P6u6rmhILNMQHaQSHqmNSiDJeA0MzMTc87 u8vxmJmRnLsk88uYIMMz6w ).

3.JAYDIP DATTA, IMA n.a. M.D. Shapovalenko and the scientific journal "Mariinskaya Academy" linked research : INDUSTRIAL ENGINEERING OR ENGINEER IN INDUSTRIES: A NOTE ,November 2019 ,DOI: 10.13140/RG.2.2.28166.93768

https://www.researchgate.net/publication/337631834 IMA na MD Shapovalenko and the sci entific journal Mariinskaya Academy linked research INDUSTRIAL ENGINEERING OR EN GINEER IN INDUSTRIES A NOTE? sg\%5B0\%5D=kyIrU8r99ggRVSp6aoT26QStyM1Mf6PU yJp7eqcvsuZUVW7bCEhWb2HpEwTwjRZK3IvPOVDwv4nh6dtinMKb 92pe-AHc9-

ai8hwkiGg.uboBuoGs7usGB4OdaBbj3Lc8YJO3ve5IGmgMxZq7bnl07YjcakufXjBedTojMRaqf3e IHPBjO9MIDX7ogv8GJg ).

4.JAYDIP DATTA, International Mariinskaya Academy n.a. M.D. Shapovalenko and the scientific journal "Mariinskaya Academy" linked research : MEMBERSHIP OF HONOUR ( Indian Institute of Chemical Engineers ) IIChE , 2012 ,November 2019 ,DOI:

10.13140/RG.2.2.31682.32966/2 .

https://www.researchgate.net/publication/337427406 IMA named after MD Shapovalenko In dustrial Engineering 24 linked Research CHEMICAL ENGINEERING An INDUSTRIAL RE VIEW REVISED on 0912 2019? sg\%5B0\%5D=Txnu2NaDOl8587ygpjySUPsh3haxloXrrzQt EPIXxZ2wkw5nJmNgQWgg1rgUtyandG3mv5MaotbdMRdIXdLoU-B-

7ivRcCceh40F4pyV.4zr PlgNCaum8gRjO7OvNXo9j6ISLxzzICZLGta9TWCJWAgFISA8FRYuy UiVkMEfCn9vzAxwfDdj 67nlyB2xA .

5.JAYDIP DATTA, Industrial engineering: A Chapter ,March 2020

DOl: 10.13140/RG.2.2.26142.46407.

(https://www.researchgate.net/publication/339828291 Industrial engineering A Chapter? s $\mathrm{g}=$ ayqzGEfk1G 6NbyYGd-RdMHQ2HqsvoEz4C-

oBvQ5ggd4rbMcRfNQhfEVVZbvshuR bwHA21sy8ic4MljMdWTiX1x8oJO4XX166zS w30.u 4fM2JC9gnBMRFWN3wYRuSc5dcKzuNIIZ69BIVLEef6M23j4r7pu2yQtcO7UmbkOcbjiRvHT Q14xWzmYY-irwA ). 

CONCLUSION: In the vast area of Engineering Research \& Technology three main thrust areas are highlighted with FULL TEXT LINK for detail \& elaborative discussion of each areas. 